\title{
Electronic hole transfer in rutile and anatase TiO2: Effect of a delocalization error in the density functional theory on the charge transfer barrier height
}

\author{
Zawadzki, Pawel; Rossmeisl, Jan; Jacobsen, Karsten Wedel
}

Published in:

Physical Review B Condensed Matter

Link to article, DOI:

10.1103/PhysRevB.84.121203

Publication date:

2011

Document Version

Publisher's PDF, also known as Version of record

Link back to DTU Orbit

Citation (APA):

Zawadzki, P., Rossmeisl, J., \& Jacobsen, K. W. (2011). Electronic hole transfer in rutile and anatase TiO2: Effect of a delocalization error in the density functional theory on the charge transfer barrier height. Physical Review $B$ Condensed Matter, 84(12), 121203. https://doi.org/10.1103/PhysRevB.84.121203

\section{General rights}

Copyright and moral rights for the publications made accessible in the public portal are retained by the authors and/or other copyright owners and it is a condition of accessing publications that users recognise and abide by the legal requirements associated with these rights.

- Users may download and print one copy of any publication from the public portal for the purpose of private study or research.

- You may not further distribute the material or use it for any profit-making activity or commercial gain

- You may freely distribute the URL identifying the publication in the public portal 


\title{
छs \\ Electronic hole transfer in rutile and anatase $\mathrm{TiO}_{2}$ : Effect of a delocalization error in the density functional theory on the charge transfer barrier height
}

\author{
Paweł Zawadzki, Jan Rossmeisl, and Karsten Wedel Jacobsen \\ Department of Physics, Center for Atomic-Scale Materials Design, Technical University of Denmark, DK-2800 Kgs. Lyngby, Denmark
}

(Received 1 August 2011; published 29 September 2011)

\begin{abstract}
We analyze the deformation of the potential energy surface (PES) due to the incorrect description of fractional electron systems (the nonlinearity of the energy with electron number) within a (semi)local density functional theory (DFT). Particularly sensitive to this failure are polaronic systems where charge localization is strongly coupled to lattice distortion. As an example we calculate the adiabatic PES for the hole transfer process in rutile and anatase $\mathrm{TiO}_{2}$. (Semi)local DFT leads to qualitatively wrong, barrierless curves. Removal of the nonlinearity improves the PES shape and allows us to calculate hole mobilities.
\end{abstract}

DOI: 10.1103/PhysRevB.84.121203

PACS number(s): 72.20.Ee, 71.38.Ht, 71.15.Mb, 81.05.Hd

Introduction. Inconsistent treatment of states with different degrees of localization is a known failure of standard (semi)local density functional theory (DFT). A clear case is the dissociation of $X_{2}{ }^{+}$systems, such as $\mathrm{He}_{2}{ }^{+}$, for which the solutions $X^{\lambda}-X^{1-\lambda}$ should be degenerated for $0 \leqslant \lambda \leqslant$ 1 at infinite separation whereas (semi)local DFT favors energetically homolytic $X^{+0.5}-X^{+0.5}$ dissociation. ${ }^{1}$ This bias can be traced back to the behavior of fractional electron systems. The exact energy is linear as the number of electrons is varied between integers. ${ }^{2}$ (Semi)local approximations are $\delta N$ convex, thus solutions with electron density delocalized over several fragments are artificially favored over those having an electron localized on one fragment. ${ }^{1,3,4}$ This biased behavior is related to the spurious electron self-interaction present in the common functionals. The relation, however, is by far not a straightforward one as it has been shown that even one-electron self-interaction free methods fail to reproduce linearity of the energy as a function of electron number. ${ }^{1,3}$

The inconsistent description of states with different degrees of localization is especially troublesome in polaronic systems where the charge localization is coupled to lattice distortion. ${ }^{5,6}$ (Semi)local DFT artificially elevates areas on the potential energy surface (PES) related to more localized states, therefore it provides too delocalized solutions and incorrect atomic structures. Such PES deformations lead to incorrect pictures of processes where the degree of localization changes. Charge transfer is one of these processes as states along a charge transferring coordinate vary in the amount of charge sharing between the transferring sites. Here we analyze the PES arising from the electronic hole transfer in a technologically important photocatalyst-titanium dioxide. Transport of photogenerated holes to surfaces is a necessary step preceding photocatalytic reaction. In this material hole transfer largely determines photocatalytic efficiency as charge recombination in the bulk and reactions on the surface occur on similar time scales. ${ }^{7}$ Various experimental techniques such as electron paramagnetic resonance, ${ }^{8-10}$ transient absorption spectroscopy, ${ }^{11-16}$ and photoluminescence ${ }^{17-19}$ have revealed that photogenerated holes are trapped. Yet the nature of the trapping sites has remained obscure. Only recently it is becoming evident that holes self-trap intrinsically, both in rutile ${ }^{8,20}$ and in anatase, ${ }^{20}$ forming $\mathrm{O}^{-}$small polarons. $^{21}$
Model. To examine the effect of the $\delta N$ convexity of (semi)local functionals on the PES for the self-trapped hole transfer, we first introduce a two-site model of the process. The hole is transferred between lattice sites $L$ and $R: L^{\lambda}+R \rightarrow$ $L+R^{\lambda}$, where the hole number can be fractional, $\lambda \in[0,1]$. We assume that the system interacts with the lattice via a single mode $x$ and at $\lambda=0$ the PES is harmonic, $E(x, \lambda=$ $0)=\frac{1}{2} K x^{2}$. In the basis of orthonormal states localized on these sites, the electronic Hamiltonian takes the form

$$
H_{e}=\left(\begin{array}{cc}
\varepsilon_{L}+V x & t \\
t & \varepsilon_{R}-V x
\end{array}\right) .
$$

$\varepsilon_{L}, \varepsilon_{R}$ are the on-site energies associated with the basis functions $|L\rangle$ and $|R\rangle ; t$ is the electronic coupling between the sites; and $V$ is the strength of the electron-mode interaction.

In the static approximation-excluding the mode kinetic energy - the hole transfer occurs on a single PES. We construct the PES by removing a fraction $\lambda$ of a hole from the highest occupied eigenstate $\varepsilon$ of $H_{e}$ :

$$
\Delta E(x, \lambda)=\frac{1}{2} K x^{2}-\lambda \varepsilon(x) .
$$

For $L$ and $R$ representing $\mathrm{O}^{2-}$ lattice ions, $\varepsilon$ is the antibonding solution of $H_{e}$ :

$$
\varepsilon(x)=\left\{\varepsilon_{L}+\varepsilon_{R}+t \sqrt{\tan [2 \zeta(x)]^{-2}+1}\right\} / 2,
$$

where $2 \zeta(x)=\arctan \left[2 t /\left(\varepsilon_{L}-\varepsilon_{R}+2 V x\right)\right]$. In the following we consider $x \leqslant 0$; for $x>0$ indexes $L$ and $R$ should be interchanged.

The hole then occupies the antibonding state $|\Psi\rangle=$ $|L\rangle \cos [\zeta(x)]+|R\rangle \sin [\zeta(x)]$. If $\zeta(x)=0$, the hole is confined to the $L$ site; if $\zeta(x)=\pi / 4$, the hole is equally shared between the two sites-therefore $\zeta(x)$ defines the degree of hole localization. In the absence of electron-mode coupling, $\zeta$ is independent of the coordinate $x$. The composition of eigenstates then does not change along the coordinate; the PES should not be deformed if there is any bias toward a more localized or delocalized state. Contrary, for a nonzero $V$ the states along $x$ differ in the degree of localization and their inconsistent treatment will result in a deformed PES.

A $\delta N$ convexity of a (semi)local DFT leads to such inconsistent descriptions. For a quadratic behavior of the total energies of subsystems $L$ and $R$, the on-site energies vary linearly with their occupations: $\varepsilon_{L}^{\prime}(\zeta)=\varepsilon_{L}-\alpha \lambda \cos ^{2}(\zeta)$ and 


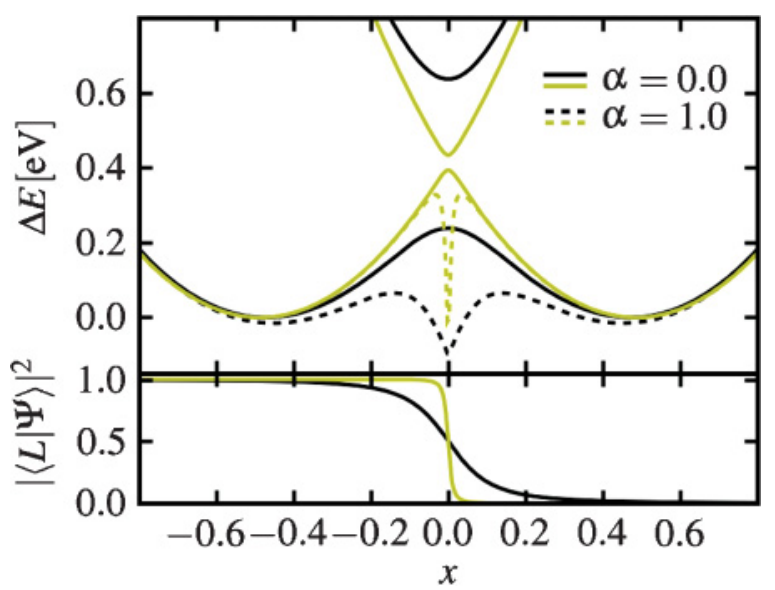

FIG. 1. (Color online) Top: The PESs for a two-state model of a hole transfer between equivalent fragments (full lines) and the deformed PESs due to $\delta N$ convexity of the energy (dashed lines). Bottom: The hole localization on the $L$ site. Curves are plotted for $t=0.2 \mathrm{eV}$ (black) and $t=0.02 \mathrm{eV}$ (yellow/gray) with $V=1.7$, $K=3.5 \mathrm{eV}$ corresponding to the path $E$ in rutile [see Fig. 2 b]. Zero energy is at the minimum of the nondeformed PES and the deformed PES is aligned at $|x| \rightarrow \infty$.

$\varepsilon_{R}^{\prime}(\zeta)=\varepsilon_{R}-\alpha \lambda \sin ^{2}(\zeta), \alpha>0$. In Fig. 1 we show the effect of such a quadratic deviation on the PES when sites $L$ and $R$ are equivalent. For $\alpha=0$ the PES exhibits a barrier at $x=0$; for $\alpha=1$ the PES shape is deformed around the transition point. The deformation increases with stronger electronic coupling $t$ as the variation of the degree of localization extends toward larger $|x|$.

A non-self-consistent (preserving the site occupations) firstorder expansion in $\alpha$ of the deformed PES yields

$$
\Delta E^{\prime}(x, \lambda) \approx \Delta E(x, \lambda)+\alpha \lambda^{2} f[\zeta(x)],
$$

where $f(\zeta)=\left\{1+\left[2 \cos ^{2}(\zeta)-1\right] / \sqrt{\tan (2 \zeta)^{2}+1}\right\} / 2$, The correction to the PES of the system with a single hole is then $\alpha f(x)$ and can be calculated as a quadratic nonlinearity of $\Delta E^{\prime}(x, \lambda)$. As one could expect, the correction is twice as large for the localized hole, $f(\zeta=0)=1$, compared to the hole shared between two sites, $f(|\zeta| \rightarrow \pi / 4)=\frac{1}{2}$.

Computational details. We perform revised Perdew-BurkeErnzerhof (RPBE) DFT ${ }^{22}$ calculations within the projector augmented-wave formalism implemented in the GPAW code. ${ }^{23}$ The wave functions, densities and potentials are described on a grid with a spacing of $0.2 \AA$. Atomic structures are defined by lattice vectors $\mathbf{a}^{\prime}=3(\mathbf{a}+\mathbf{b}), \mathbf{b}^{\prime}=2(\mathbf{a}-\mathbf{b}), \mathbf{c}^{\prime}=2 \mathbf{c}$ for rutile, and $\mathbf{a}^{\prime}=3 \mathbf{a}, \mathbf{b}^{\prime}=3 \mathbf{b}, \mathbf{c}^{\prime}=2 \mathbf{c}$ for anatase, $\mathbf{a}, \mathbf{b}$, and $\mathbf{c}$ being the vectors of the respective tetragonal crystallographic cells. ${ }^{20,24}$ The Brillouin zone is sampled on $3 \times 2 \times 2$ and $2 \times 2 \times 1$ Monkhorst-Pack meshes for rutile and anatase, respectively.

The basic, oxygen-based, building motif of the anatase and rutile structures is a flat $C_{2 v}$-symmetric $\mathrm{OTi}_{3}$ unit. Upon hole localization the three OTi bonds elongate by 0.1$0.2 \AA$, preserving the initial symmetry. ${ }^{20}$ The hole occupies a $p$-like orbital centered on the oxygen site and is perpendicular to the $\mathrm{OTi}_{3}$ plane. In anatase such distortion renders the localized hole state more stable than the delocalized one, whereas in rutile the two have a comparable stability. ${ }^{20} \mathrm{We}$

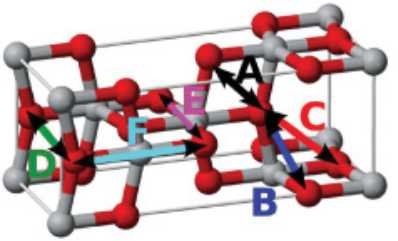

(a) Anatase

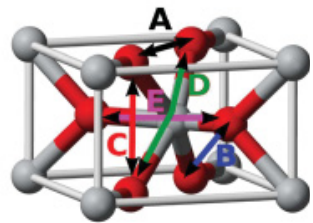

(b)Rutile
FIG. 2. (Color online) Hole transfer paths between neighboring oxygen lattice sites in $\mathrm{TiO}_{2}$.

consider hole transfer between two neighboring oxygen sites $L$ and $R$. There are several such possible pairs of oxygen atoms (see Fig. 2). For each pair we construct a set of structures $\mathbf{Q}$ defined through linear interpolation between the distortions localized on the two sites $\mathbf{Q}_{\mathbf{L}}$ and $\mathbf{Q}_{\mathbf{R}}$, respectively: $\mathbf{Q}=\left[x\left(\mathbf{Q}_{\mathbf{R}}-\mathbf{Q}_{\mathbf{L}}\right)+\mathbf{Q}_{\mathbf{R}}+\mathbf{Q}_{\mathbf{L}}\right] / 2$.

For the constructed paths, we then calculate a set of PESs, $\Delta E^{\prime}(x, \lambda)$, by removing a fraction of an electron $\lambda$ from the highest occupied state and placing it at the bottom of the conduction band using linear expansion $\triangle$-SCF (delta selfconsistent field). ${ }^{20,25,26}$ To correct the PESs for the spurious delocalization error, we remove the PES quadratic nonlinarlity in $\lambda: \Delta E(x, \lambda=1)=\Delta E^{\prime}(x, \lambda=1)-\alpha f(x)$.

Results and discussion. In the top plots of Fig. 3 we show PESs resulting from RPBE DFT calculations for different paths. The curves are seen to exhibit a drop around the transition point. We have shown that this characteristic kink is caused by the $\delta N$ convexity of (semi)local functionals. At $x=0$ the delocalization of the hole charge is the largest - the hole is shared between two sites; therefore the relative error with respect to the localized state takes its maximum. The quadratic nonlinearity of $\Delta E^{\prime}(x, \lambda), \alpha f$, is shown in the middle plots of Fig. 3. The two-state model predicts that at large $|x|$ the correction should be twice as big as at $x=0$. This ratio is not strictly conserved for some paths. The hole orbital

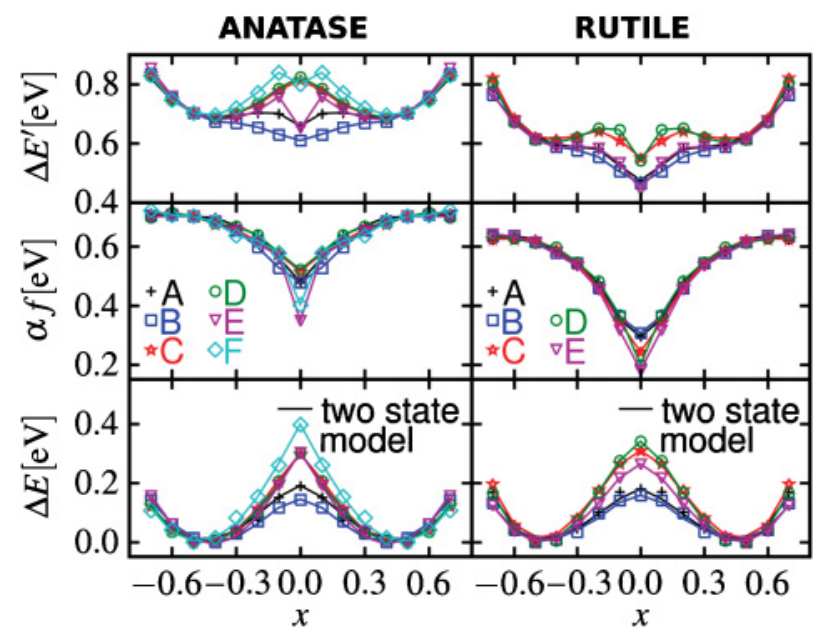

FIG. 3. (Color online) Top: PESs $\Delta E^{\prime}(x, \lambda=1)$ resulting from RPBE DFT calculations are plotted for different hole transfer paths. Middle: The nonlinearity of PES $\alpha f(x)$ with respect to hole number $\lambda$. Bottom: The corrected PES, $\Delta E(x, \lambda=1)=\Delta E^{\prime}(x, \lambda=1)-$ $\alpha f(x)$. 
hybridizes with the surrounding orbitals, therefore the degree of localization can to some extent be different than in the model. In the bottom plots of Fig. 3 we show the corrected PESs, $\Delta E(x, \lambda=1)$. Their shape is improved, notably the kinks around $x=0$ are removed.

We use the thus corrected PESs to calculate hightemperature hole mobility. At high $T$ small-polaron transport is dominated by the hopping mechanism. If the relative dynamics of the hole is fast compared to lattice fluctuations, the hole instantaneously follows the lattice coordinate and the charge transfer proceeds via barrier crossing at the transition point - the adiabatic limit. The hopping frequency in this case is given by ${ }^{27}$

$$
k=[\Omega /(2 \pi)] \exp \left[-E_{b}^{\mathrm{ad}} /\left(k_{B} T\right)\right],
$$

where $E_{b}^{\text {ad }}$ is the adiabatic barrier height and $\Omega$ is the transferring mode frequency. In the nonadiabatic regime the hole is not able to follow the distortion. The transfer between two sites occurs at the intersection of two nonadiabatic PESs with a certain transition probability. The resulting hopping frequency in this case is given by ${ }^{27}$

$$
k=t^{2} \sqrt{\pi /\left(4 \hbar^{2} E_{b}^{\mathrm{nad}} k_{B} T\right)} \exp \left[-E_{b}^{\mathrm{nad}} /\left(k_{B} T\right)\right],
$$

where $E_{b}^{\mathrm{nad}}=E_{b}^{\mathrm{ad}}+t$ is the nonadiabatic barrier height and $t$ is the electronic coupling. We discriminate between these cases by calculating the parameter $\eta_{2}=t^{2} /\left(\hbar \Omega \sqrt{E^{\text {nad }} k T}\right)$ describing the relative inertia of the nuclear and electronic motion. ${ }^{27}$ For $\eta_{2}>1$ we assume adiabatic transport and otherwise the nonadiabatic one. At $1300 \mathrm{~K}$ we find all the nearest paths in rutile and paths $A$ and $B$ in anatase adiabatic. Apart from paths $C$ and $E$ in rutile, this is in accordance with wave-function calculations ${ }^{28}$ (our notation $A B C \ldots$ corresponds to $D F B C E A$ and $B C A D E$ in Ref. 28 for anatase and rutile, respectively).

We calculate the drift mobility using the EinsteinSmoluchowski relation $\mu=e D /\left(k_{B} T\right) . D$ is the average hole diffusion coefficient $D=\sum_{i \in \mathrm{A} \ldots} n_{i} k_{i}\left|\mathbf{R}_{\mathbf{i}}\right|^{2} / 3$, where $n_{i}$ is a number of equivalent paths, $k_{i}$ is the transfer frequency, and $\left|\mathbf{R}_{\mathbf{i}}\right|$ is the transfer distance. The two-site model for small-polaron hopping holds if $\eta_{1}=t / E_{b}^{\text {nad }} \ll 1 .^{27}$ For anatase we find $\eta_{1} \in(0.05,0.56) \mathrm{eV}$ and for rutile $\eta_{1} \in(0.27$, $0.67) \mathrm{eV}$. Smaller $\eta_{1}$ in anatase indicates that the hole in this phase is more localized. (For what is also reflected in larger nonlinearities $\alpha f$, see the middle plots in Fig. 3.)

The characteristic parameters of the different hole transfer paths in anatase and rutile are shown in the Supplemental Material. ${ }^{29} \mathrm{We}$ only note here that $\hbar \Omega(\approx 20 \mathrm{meV}$ in rutile and $\approx 40 \mathrm{meV}$ in anatase) is much lower than the highest-frequency mode at $\sim 100 \mathrm{meV}$ used to calculate hole mobility in Ref. 28 . We find our values in accordance with the fact that the transfer coordinate arises mostly from the displacements of the cationic sublattice corresponding to the breathing mode of the flat
$\mathrm{OTi}_{3}$ unit. Such vibrations resemble the optical modes $B_{1 g}$ at $50 \mathrm{meV}$ and $64 \mathrm{meV}$ in anatase ${ }^{30}$ and $B_{1 u}$ at $14 \mathrm{meV}$ and $50 \mathrm{meV}$ in rutile. ${ }^{31}$

In units of $\mathrm{cm}^{2} \mathrm{~V}^{-1} \mathrm{~s}^{-1}$, mobilities measured in electrical conductivity experiments at $1300 \mathrm{~K}$ are 0.25 (Ref. 32) for single crystal and $0.16,^{32} 5.0 \times 10^{-2}$ (Ref. 33) for polycrystalline samples. At this temperature we find hole mobilities of $5.2 \times 10^{-2}$ and $4.0 \times 10^{-2}$ for anatase and rutile, respectively. At $873 \mathrm{~K}$ the transient grating technique predicts a value of $0.18,{ }^{34}$ whereas we find $3.3 \times 10^{-2}$ for anatase and $3.1 \times 10^{-2}$ for rutile.

Clearly, the calculated values underestimate the hole mobility. A better guess for the transition points (achieved by constraining the hole to be equally shared between the hole transferring sites) lowers the adiabatic barriers from $E_{\text {ad }} \in(0.14,0.39)$ to $E_{\text {ad }} \in(0.09,0.39) \mathrm{eV}$ in anatase and from $E_{\text {ad }} \in(0.14,0.29)$ to $E_{\text {ad }} \in(0.06,0.21) \mathrm{eV}$ in rutile, whereas only barrierless paths could provide mobilities close to the experimental ones.

This underestimation is likely due to the contribution of valence-band (VB) holes to the hole conduction mechanism. At $1300 \mathrm{~K}$-assuming hole stabilization of $0.05 \mathrm{eV}$ in rutile and $0.2 \mathrm{eV}$ in anatase $\mathrm{e}^{20} \sim 23 \%$ and $\sim 14 \%$ of the total hole concentration occupies the VB in rutile and anatase, respectively. Two types of hole transport mechanisms are more evident at lower temperatures, where the disparity between the band and small-polaron mobilities increases (the latter becomes relatively immobile). Room-temperature experiments indicate the existence of two hole dynamics time scales: a fast one, after excitation-hole transfer to the surface within $\tau \approx r^{2} D /(k T)$, where $r$ is the particle diameter and $D$ corresponds to the VB hole diffusion coefficient; and a much slower, nanosecond-microsecond decay of surface trapped holes. ${ }^{11-19}$ We think that these two time scales are related to the VB band and the small-polaron transport mechanisms.

Conclusion. In summary, we have shown that the spurious nonlinearity of the total energy in a (semi)local DFT leads to a significant deformation of the PES if the states on the PES differ in the degree of charge localization. As an example we studied the electronic hole hopping in $\mathrm{TiO}_{2}$. (Semi)local DFT results in a quantitatively incorrect barrierless PES. By removing the energy nonlinearity in the fractional hole number from the PES, we improved the PES shape. With the thus corrected PES we calculated high-temperature hole hopping mobilities. Our results indicate that both the trapped and the VB holes contribute to the hole transport mechanism.

CAMD is funded by the Lundbeck foundation. This work was supported by the Danish Center for Scientific Computing. Support from the Danish Council for Technology and Innovation's FTP program and the Danish Council for Strategic Research though the HyCycle Center (No. 2104-07-0041) is acknowledged.

\footnotetext{
*zawpaw@fysik.dtu.dk

${ }^{1}$ A. Ruzsinszky, J. P. Perdew, G. I. Csonka, O. A. Vydrov, and G. E. Scuseria, J. Chem. Phys. 126, 104102 (2007).
}

${ }^{2}$ J. F. Janak, Phys. Rev. B 18, 7165 (1978).

${ }^{3}$ A. Ruzsinszky, J. P. Perdew, G. I. Csonka, O. A. Vydrov, and G. E. Scuseria, J. Chem. Phys. 125, 194112 (2006). 
${ }^{4}$ J. P. Perdew, A. Ruzsinszky, G. I. Csonka, O. A. Vydrov, G. E. Scuseria, V. N. Staroverov, and J. Tao, Phys. Rev. A 76, 040501 (2007).

${ }^{5}$ J. L. Gavartin, P. V. Sushko, and A. L. Shluger, Phys. Rev. B 67, 035108 (2003).

${ }^{6}$ S. Lany and A. Zunger, Phys. Rev. B 80, 085202 (2009).

${ }^{7}$ A. Fujishima, X. Zhang, and D. A. Tryk, Surf. Sci. Rep. 63, 515 (2008).

${ }^{8}$ S. Yang, A. T. Brant, and L. E. Halliburton, Phys. Rev. B 82, 035209 (2010).

${ }^{9}$ O. I. Micic, Y. N. Zhang, K. R. Cromack, A. D. Trifunac, and M. Thurnauer, J. Phys. Chem. 97, 7277 (1993).

${ }^{10}$ R. F. Howe and M. Gratzel, J. Phys. Chem. 91, 3906 (1987).

${ }^{11}$ T. Yoshihara, R. Katoh, A. Furube, Y. Tamaki, M. Murai, K. Hara, S. Murata, H. Arakawa, and M. Tachiya, J. Phys. Chem. B 108, 3817 (2004).

${ }^{12}$ Y. Tamaki, A. Furube, R. Katoh, M. Murai, K. Hara, H. Arakawa, and M. Tachiya, C. R. Chim. 9, 268 (2006).

${ }^{13}$ D. Bahnemann, M. Hilgendorff, and R. Memming, J. Phys. Chem. B 101, 4265 (1997).

${ }^{14}$ A. Furube, T. Asahi, H. Masuhara, H. Yamashita, and M. Anpo, J. Phys. Chem. B 103, 3120 (1999).

${ }^{15}$ Y. Tamaki, A. Furube, M. Murai, K. Hara, R. Katoh, and M. Tachiya, Phys. Chem. Chem. Phys. 9, 1453 (2007).

${ }^{16}$ X. Yang and N. Tamai, Phys. Chem. Chem. Phys. 3, 3393 (2001).

${ }^{17}$ L. Cavigli, F. Bogani, A. Vinattieri, V. Faso, and G. Baldi, J. Appl. Phys. 106, 053516 (2009).

${ }^{18}$ N. Harada, M. Goto, K. Iijima, H. Sakama, N. Ichikawa, H. Kunugita, and K. Ema, Jpn. J. Appl. Phys. 1 46, 4170 (2007).
${ }^{19}$ K. Fujihara, S. Izumi, T. Ohno, and M. Matsumura, J. Photochem. Photobiol. B 132, 99 (2000).

${ }^{20}$ P. Zawadzki, K. W. Jacobsen, and J. Rossmeisl, Chem. Phys. Lett. 506, 42 (2011).

${ }^{21}$ O. F. Schirmer, J. Phys. Condens. Matter 18, R667 (2006).

${ }^{22}$ B. Hammer, L. B. Hansen, and J. K. Norskov, Phys. Rev. B 59, 7413 (1999).

${ }^{23}$ J. Enkovaara et al., J. Phys. Condens. Matter 22, 253202 (2010).

${ }^{24}$ J. I. Martinez, H. A. Hansen, J. Rossmeisl, and J. K. Norskov, Phys. Rev. B 79, 045120 (2009).

${ }^{25}$ J. Gavnholt, T. Olsen, M. Engelund, and J. Schiotz, Phys. Rev. B 78, 075441 (2008).

${ }^{26}$ R. O. Jones and O. Gunnarsson, Rev. Mod. Phys. 61, 689 (1989).

${ }^{27} \mathrm{H}$. Bottger and V. V. Bryksin, Hopping Conduction in Solids $(\mathrm{VCH}$, Berlin, 1985).

${ }^{28}$ N. A. Deskins and M. Dupuis, J. Phys. Chem. C 113, 346 (2009).

${ }^{29}$ See Supplemental Material at http://link.aps.org/supplemental/ 10.1103/PhysRevB.84.121203 for the characteristic parameters of the different hole transfer paths in anatase and rutile.

${ }^{30}$ T. Ohsaka, F. Izumi, and Y. Fujiki, J. Raman Spectrosc. 7, 321 (1978).

${ }^{31}$ J. G. Trayler, H. G. Smith, R. M. Nicklow, and M. K. Wilkinson, Phys. Rev. B 3, 3457 (1971).

${ }^{32}$ T. Bak, M. K. Nowotny, L. R. Sheppard, and J. Nowotny, J. Phys. Chem. C 112, 12981 (2008).

${ }^{33}$ U. Balachandran and N. Eror, J. Mater. Sci. 23, 2676 (1988).

${ }^{34}$ S. Nakabayashi, S. Komuro, Y. Aoyagi, and A. Kira, J. Phys. Chem. 91, 1696 (1987). 